\title{
Fixed points of multi-valued monotone operators and the solvability of a fractional integral inclusion
}

\author{
Yuqiang Feng ${ }^{1,2^{*}}$ and Yuanyuan Wang ${ }^{1,2}$
}

\section{"Correspondence:}

yafeng6@126.com

'school of Science, Wuhan

University of Science and

Technology, Wuhan, 430065,

P.R. China

${ }^{2}$ Hubei Province Key Laboratory of

Systems Science in Metallurgical

Process, Wuhan, 430065, P.R. China

\section{严 Springer}

\begin{abstract}
Based on the characterizations of reproducing cones, some fixed point theorems for multi-valued increasing, decreasing, and mixed monotone operators are established. As an application, the solvability of a fractional integral inclusion is discussed.
\end{abstract}

Keywords: reproducing cone; multi-valued operator; fixed point; fractional integral inclusion; monotone

\section{Introduction}

Single-valued monotone operators have been widely investigated. The results on the existence of fixed points for single-valued monotone operators are bounteous and successful and have found various applications to nonlinear integral equations and differential equations. For details, we can refer to [1-4] and the references therein.

It is well known that mixed monotone operators were introduced by Guo and Lakshmikantham [5] in 1987. Later, Bhaskar and Lakshmikantham [6] introduced the notation of coupled fixed point and proved some coupled fixed point results under certain conditions, in a complete metric space endowed with a partial order. Their study not only has important theoretical meaning but also wide applications in engineering, nuclear physics, biological chemistry technology, economics, etc. (see [5-13] and the references therein).

Very recently, Harjani, Lopez and Sadarangani [14] have established the existence results of coupled fixed point for mixed monotone operators and further obtained their applications to integral equations. Then in [15], Bu, Feng, and Li extended the study to mixed monotone ternary operators.

It is natural to extend these studies to the multi-valued case. In 1984, Nishniannidze [16] introduced the multi-valued monotone operators. Nguyen and Nguyen [17] investigated the fixed points for multi-valued increasing operators and then in [18], a fixed point theorem for a multi-valued increasing operator was established and applied to a discontinuous elliptic equation. Huang and Fang [19] extended the mixed monotone operators to the multi-valued case, the obtained result was applied to a class of integral inclusions. For some recent fixed point theorems for a multi-valued monotone operator, a mixed monotone operator, and their applications in differential equations and differential inclusions, 
we can refer to [19-25] and the references therein for details. All these papers need the existence of a lower or an upper solution to the operator inclusion.

Motivated by the above work, in this paper, we shall use the properties of reproducing cones to establish some fixed point theorems for multi-valued monotone and mixed monotone operators. Compared with the previous work, we remove the requirement of the existence of a lower or upper solution. When the operator is single-valued and mixed monotone, we get an existence and uniqueness result.

This paper is organized as follows: in Section 2, some basic knowledge and the properties of a reproducing cone are presented; then in Section 3, the existence of a fixed point for multi-valued monotone operators is established; in Section 4, coupled fixed point theorems are presented for single-valued and multi-valued mixed monotone operators. In the final section, as applications of our results, the solvability of a fractional integral inclusion is discussed.

\section{Preliminaries}

In this section, we recall some standard definitions and notations needed in the following section. For convenience of the reader, we suggest that one refers to $[3,26]$ for details.

At the beginning of this section, let us recall some concepts of the theory of cones in Banach spaces. These concepts play an important role in the remainder of this paper.

Let $X$ be a Banach space, a closed convex set $P \subset X$ is called a cone, if $x \in P$ and $x \neq 0$ implies $\alpha x \in P$ for $\alpha \geq 0$ and $\alpha x \notin P$ for $\alpha<0$. A cone defines a partial order in the Banach space $X$ : we write $x \leq y$ or $y \geq x$ if $y-x \in P$. The relation enjoys the following properties: inequalities may be multiplied by a nonnegative numbers; inequalities of the same kind may be added by terms; one may pass to the limit in inequalities; $x \leq y$ and $y \leq x$ implies $x=y$. Denote $-P=\{-x \mid x \in P\}$, then $-P$ is a cone too.

It is well known that if $X$ be a partially ordered Banach space endowed with partial order $\leq$, then the subset $P=\{x \in X \mid 0 \leq x\}$ is a cone.

Definition 2.1 [26] A cone $P$ is called normal, if there is a $K>0$, such that $0 \leq x \leq y$ implies $\|x\| \leq K\|y\|$ and $K$ does not depend on $x$ and $y$. Any such $K=K(P)$ is called a normal constant of $P$. A cone $P$ is called reproducing, if each $x \in X$ admits a presentation $x=u-v(u, v \in P)$. The elements $u, v$ are, of course, not unique.

For the details of cone theory, see [26] and references therein.

Lemma 2.2 [4] Let $X$ be a Banach space, $P \subset X$ be a cone. The following assertions are equivalent:

(1) $P$ is reproducing;

(2) every pair $x, y \in X$ has a lower bound;

(3) every pair $x, y \in X$ has an upper bound;

(4) $\forall x \in X$, there exists $u \geq 0$ such that $x \leq u$;

(5) $\forall x \in X$, there exists $u \leq 0$ such that $x \geq u$.

Definition 2.3 [20] Let $X$ be a topology space, ' $\leq$ ' be a partial order endowed on $X$, let $A$, $B$ be two nonempty subsets of $X$, the relations between $A$ and $B$ are defined as follows:

(1) $A \prec_{1} B$ if for every $a \in A$, there exists $b \in B$ such that $a \leq b$;

(2) $A \prec_{2} B$ if for every $b \in B$, there exists $a \in A$ such that $a \leq b$;

(3) $A \prec B$ if $A \prec_{1} B$ and $A \prec_{2} B$. 
Definition 2.4 Let $(X, \leq)$ be an ordered Banach space, the partial order ' $\leq$ ' is induced by cone $P$.

(1) A multi-valued operator $T: X \rightarrow 2^{X} \backslash\{\phi\}$ is called increasing, if for $\forall x, y \in X, x \leq y$ implies $T(x) \prec T(y)$.

(2) A multi-valued operator $T: X \rightarrow 2^{X} \backslash\{\phi\}$ is called decreasing, if for $\forall x, y \in X, x \leq y$ implies $T(y) \prec T(x)$.

(3) A multi-valued operator $T: X \times X \rightarrow 2^{X} \backslash\{\phi\}$ is called mixed monotone, if for $\forall x_{1}, x_{2}, y_{1}, y_{2} \in X, x_{1} \leq x_{2}, y_{2} \leq y_{1}$ implies $T\left(x_{1}, y_{1}\right) \prec T\left(x_{2}, y_{2}\right)$.

\section{Definition 2.5}

(1) Let $T: X \rightarrow 2^{X} \backslash\{\phi\}$ be a multi-valued operator, $\bar{x} \in X$ is called a fixed point of $T$, if $\bar{x} \in T(\bar{x})$.

(2) Assume $T: X \times X \rightarrow 2^{X} \backslash\{\phi\}$ is a multi-valued operator, $(\bar{x}, \bar{y}) \in X \times X$ is called a coupled fixed point of $T$, if $\bar{x} \in T(\bar{x}, \bar{y})$ and $\bar{y} \in T(\bar{y}, \bar{x})$.

\section{Fixed points for multi-valued monotone operators}

In this section, some fixed point theorems for multi-valued increasing and decreasing operators are proved in partial ordered Banach space.

Throughout this paper, we assume that $X$ is a Banach space, $P$ is a normal and reproducing cone in $X$ and the partial order ' $\leq$ ' is induced by the cone $P$.

Theorem 3.1 Suppose the multi-valued operator $T: X \rightarrow 2^{X} \backslash\{\phi\}$ satisfies the following conditions:

(1) For any $x \in X, T(x)$ is a nonempty and closed subset of $X$.

(2) There exists a linear operator $L: X \rightarrow X$ with spectral radius $r(L)<1, L(P) \subset P$ such that, for any $x, y \in X, x \leq y$ implies:

(i) for any $u \in T(x)$, there exists $v \in T(y)$ satisfying

$$
0 \leq v-u \leq L(y-x)
$$

(ii) for any $v \in T(y)$, there exists $u \in T(x)$ satisfying

$$
0 \leq v-u \leq L(y-x)
$$

Then $T$ has a fixed point in $X$.

Proof The proof is given in three steps.

Step 1. There exists $x_{0} \in X$, such that $\left\{x_{0}\right\} \prec_{1} T\left(x_{0}\right)$.

In fact,

(1) if $\{0\} \prec_{1} T(0)$, then $x_{0}=0$;

(2) if $\{0\} \prec_{1} T(0)$ does not hold.

Let $y_{0} \in T(0)$. Since $P$ is reproducing, by Lemma 2.2, there is $v \in(-P)$, such that $v \leq y_{0}$.

Due to the assumption $r(L)<1, L(P) \subset P$, we know that the equation

$$
(I-L) x=-v
$$

has a unique solution $z_{0} \in P$ by Banach's contraction theorem. 
Let $x_{0}=-z_{0}$, by assumption (2)(ii), for $y_{0} \in T(0)$, there exists $u_{0} \in T\left(x_{0}\right)$ such that

$$
0 \leq y_{0}-u_{0} \leq L\left(0-x_{0}\right)
$$

hence,

$$
u_{0} \geq y_{0}-L\left(z_{0}\right)
$$

Noting that $v \leq y_{0}$ and $(I-L)\left(z_{0}\right)=-v$, we have

$$
u_{0} \geq y_{0}-L\left(z_{0}\right) \geq v-L\left(z_{0}\right)=-z_{0}=x_{0},
$$

which implies $\left\{x_{0}\right\} \prec_{1} T\left(x_{0}\right)$.

Step 2. There exists an increasing and fundamental sequence $\left\{x_{n}\right\} \subset X$.

In fact, let $x_{1}=u_{0}$, due to assumption (2)(i), there exists $x_{2} \in T\left(x_{1}\right)$ such that

$$
0 \leq x_{2}-x_{1} \leq L\left(x_{1}-x_{0}\right)
$$

Repeating the arguments above for the pair $x_{1}, x_{2}$ in place of $x_{0}, x_{1}$ and so on, we can find an increasing sequence $\left\{x_{n}\right\}$ satisfying

$$
x_{n} \in T\left(x_{n-1}\right), \quad 0 \leq x_{n+1}-x_{n} \leq L^{n}\left(x_{1}-x_{0}\right) .
$$

Since $\lim _{n \rightarrow \infty}\left(\left\|L^{n}\right\|\right)^{\frac{1}{n}}=r(L)$ and $r(L)<1$, we have $\left\|L^{n}\right\| \leq q^{n}$ for some $q \in(0,1)$ and for all sufficiently large $n$.

At this moment, we get

$$
\left\|x_{n}-x_{n-1}\right\| \leq K\left\|L^{n}\right\| \cdot\left\|x_{1}-x_{0}\right\| \leq K q^{n}\left\|x_{1}-x_{0}\right\|
$$

where $K$ is the normal constant of cone $P$.

This implies $\left\{x_{n}\right\}$ is fundamental. Since $X$ is complete, there exists a unique $x^{*} \in X$ such that $x_{n} \rightarrow x^{*}$.

Step 3. $x^{*} \in T\left(x^{*}\right)$.

Since $x_{n-1} \leq x^{*}, x_{n} \in T\left(x_{n-1}\right)$, by assumption 2(i), there exists $y_{n} \in T\left(x^{*}\right)$, such that

$$
0 \leq y_{n}-x_{n} \leq L\left(x^{*}-x_{n-1}\right)
$$

Due to the fact that $P$ is normal, we have

$$
\left\|y_{n}-x_{n}\right\| \leq K\|L\| \cdot\left\|x^{*}-x_{n-1}\right\|
$$

which implies

$$
\lim _{n \rightarrow \infty} y_{n}=\lim _{n \rightarrow \infty}\left(y_{n}-x_{n}\right)+\lim _{n \rightarrow \infty}\left(x_{n}-x^{*}\right)+x^{*}=x^{*} .
$$

Noting that $T\left(x^{*}\right)$ is closed, we know $x^{*} \in T\left(x^{*}\right)$.

This ends the proof. 


\section{Remark 3.2}

1. In Theorem 3.1, the assumptions (2)(i) and (2)(ii) imply that $T(x) \prec T(y)$ for $x \leq y$, i.e. $T$ is a multi-valued increasing operator.

2. It should be noticed that Theorem 3.1 cannot ensure the uniqueness of a fixed point. For example, let $X=R,\|x\|=|x|, P=\{x \in R \mid x \geq 0\}$, then $X$ is a Banach space and $P$ a normal and reproducing cone.

Define $T: X \rightarrow 2^{X} \backslash\{\phi\}, L: X \rightarrow X$ as follows:

$$
\begin{aligned}
& T(x)=\left\{u \in X \mid \frac{1}{2} x-\frac{1}{2} \leq u \leq \frac{1}{2} x+\frac{1}{2}\right\}, \quad x \in X, \\
& L(x)=\frac{1}{2} x .
\end{aligned}
$$

All conditions of Theorem 3.1 are satisfied. It is easy to verify that the fixed point set of $T$ is the interval $[-1,1]$.

3. In the special case when $T$ is single-valued, assumption (1) of Theorem 3.1 is naturally satisfied. Assumption (2) is simplified thus: there exists a linear operator $L: X \rightarrow X$ with spectral radius $r(L)<1, L(P) \subset P$ such that

$$
T(y)-T(x) \leq L(y-x), \quad \forall x \leq y .
$$

In this case, for the fixed point of $T$ we have existence and uniqueness; see [4].

Remark 3.3 In Theorem 3.1, assumptions (1), (2) are sufficient, without one of them, the existence of a fixed point cannot be ensured. For example, let $X=R,\|x\|=|x|, P=\{x \in R \mid$ $x \geq 0\}$, then $X$ is a Banach space and $P$ a normal and reproducing cone.

1. Define $T: X \rightarrow 2^{X} \backslash\{\phi\}, L: X \rightarrow X$ as follows:

$$
\begin{aligned}
& T(x)= \begin{cases}(-1,1), & x \leq-1 \text { or } x \geq 1, \\
(-1, x) \cup(x, 1), & -1<x<1,\end{cases} \\
& L(x)=\frac{1}{2} x .
\end{aligned}
$$

Then assumption (2)(i), (2)(ii) of Theorem 3.1 are satisfied. But $T(x)$ is not closed. It is obvious that $T$ has no fixed point.

2. Define $T: X \rightarrow 2^{X} \backslash\{\phi\}$ as

$$
T(x)=[x+1, \infty)
$$

Then $T$ satisfies assumptions (1) and (2)(ii), where $L(x)=\lambda x$ for any $\lambda \in(0,1)$. But $T$ cannot satisfy assumption (2)(i). It is obvious that $T$ has no fixed point.

3. Define $T: X \rightarrow 2^{X} \backslash\{\phi\}$ as

$$
T(x)=(-\infty, x-1]
$$

Then $T$ satisfies assumptions (1) and (2)(i), where $L(x)=\lambda x$ for any $\lambda \in(0,1)$. But $T$ cannot satisfy assumption (2)(ii). It is obvious that $T$ has no fixed point. 
In the following, we give a fixed point theorem for multi-valued decreasing operator.

Theorem 3.4 Suppose the multi-valued operator $T: X \rightarrow 2^{X} \backslash\{\phi\}$ satisfies the following conditions:

(1) For any $x \in X, T(x)$ is a nonempty and closed subset of $X$.

(2) There exists a positive constant $c \in(0,1)$ such that, for any $x, y \in X, x \leq y$ implies:

(i) for any $u \in T(x)$, there exists $v \in T(y)$ satisfying

$$
-c(y-x) \leq v-u \leq 0
$$

(ii) for any $v \in T(y)$, there exists $u \in T(x)$ satisfying

$$
-c(y-x) \leq v-u \leq 0
$$

Then $T$ has a fixed point in $X$.

Proof Define a new mapping $S: X \rightarrow 2^{X} \backslash\{\phi\}$ as follows:

$$
S(x)=\left\{w \in X \mid w=\frac{c}{1+c} x+\frac{1}{1+c} u, u \in T(x)\right\},
$$

i.e.

$$
S(x)=\frac{c}{1+c} x+\frac{1}{1+c} T(x) .
$$

Then it is easy to verify that $S$ satisfies:

(1) For any $x \in X, S(x)$ is a nonempty and closed subset of $X$.

(2) For any $x, y \in X, x \leq y$ implies:

(i) for any $u \in S(x)$, there exists $v \in S(y)$ satisfying

$$
0 \leq v-u \leq \frac{c}{1+c}(y-x),
$$

(ii) for any $v \in S(y)$, there exists $u \in S(x)$ satisfying

$$
0 \leq v-u \leq \frac{c}{1+c}(y-x) .
$$

By Theorem 3.1, there exists $x^{*} \in X$ such that

$$
x^{*} \in S\left(x^{*}\right)
$$

i.e.

$$
x^{*} \in \frac{c}{1+c} x^{*}+\frac{1}{1+c} T\left(x^{*}\right),
$$

which implies

$$
x^{*} \in T\left(x^{*}\right) .
$$

This ends the proof. 


\section{Coupled fixed points for mixed monotone operators}

In this section, we shall establish the existence and uniqueness of coupled fixed point for single-valued mixed monotone operators, and then we extend the study to the multivalued case.

To verify the main results, we need the following lemma.

Lemma 4.1 If $(X,\|\cdot\|)$ is a Banach space, $P$ is a normal and reproducing cone in $X$, then

(1) $X \times X$ is a Banach space with the norm $\|(x, y)\|=\max \{\|x\|,\|y\|\}$.

(2) $P \times(-P)$ is a normal and reproducing cone in $X \times X$.

Proof We only prove assertion (2). Noting that a partial order $\lesssim$ of $X \times X$ can be induced by $P \times(-P)$, we have

$$
\left(x_{1}, y_{1}\right) \lesssim\left(x_{2}, y_{2}\right) \quad \Longleftrightarrow \quad x_{2}-x_{1} \in P, y_{2}-y_{1} \in(-P) \quad \Longleftrightarrow \quad x_{1} \leq x_{2}, y_{2} \leq y_{1} .
$$

(i) If $(0,0) \lesssim\left(x_{1}, y_{1}\right) \lesssim\left(x_{2}, y_{2}\right)$, then $0 \leq x_{1} \leq x_{2}, 0 \geq y_{1} \geq y_{2}$, or equivalently, $0 \leq-y_{1} \leq-y_{2}$. Since $P$ is normal,

$$
\left\|x_{1}\right\| \leq K\left\|x_{2}\right\|, \quad\left\|y_{1}\right\| \leq K\left\|y_{2}\right\|,
$$

where $K$ is the normal constant of $P$.

Hence, we know

$$
\left\|\left(x_{1}, y_{1}\right)\right\|=\max \left\{\left\|x_{1}\right\|,\left\|y_{1}\right\|\right\} \leq K \max \left\{\left\|x_{2}\right\|,\left\|y_{2}\right\|\right\}=K\left\|\left(x_{2}, y_{2}\right)\right\|,
$$

which implies $P \times(-P)$ is normal with normal constant $K$.

(ii) For arbitrary $(x, y) \in X \times X$, since $P$ is reproducing, there exist $u_{1}, v_{1}, u_{2}, v_{2} \in P$, such that $x=u_{1}-v_{1}, y=u_{2}-v_{2}$, or equivalently, $y=\left(-v_{2}\right)-\left(-v_{1}\right)$. Then

$$
(x, y)=\left(u_{1},-v_{2}\right)-\left(u_{2},-v_{1}\right) \in P \times(-P)-P \times(-P),
$$

which implies $P \times(-P)$ is a reproducing cone in $X \times X$.

Theorem 4.2 Suppose $T: X \times X \rightarrow X$ is a mixed monotone operator. Assume there exist two linear operators $L, S: X \rightarrow X$ with $\|L\|+\|S\|<1, L(P) \subset P, S(P) \subset P$ such that, for any $x_{1}, x_{2}, y_{1}, y_{2} \in X, x_{1} \leq x_{2}, y_{2} \leq y_{1}$

$$
T\left(x_{2}, y_{2}\right)-T\left(x_{1}, y_{1}\right) \leq L\left(x_{2}-x_{1}\right)+S\left(y_{1}-y_{2}\right)
$$

Then $T$ has a unique coupled fixed point $(\bar{x}, \bar{y})$ in $X \times X$. Moreover, for every $(x, y) \in X \times X$,

$$
\lim _{n \rightarrow \infty} T^{n}(x, y)=\bar{x}, \quad \lim _{n \rightarrow \infty} T^{n}(y, x)=\bar{y} .
$$

Proof Define a new mapping $F: X \times X \rightarrow X \times X$ as follows, for $(x, y) \in X \times X$ :

$$
F(x, y)=(T(x, y), T(y, x))
$$


Then $T$ has a coupled fixed point if and only if $F$ has a fixed point.

When $T$ is mixed monotone, then $\forall x_{1}, x_{2}, y_{1}, y_{2} \in X$,

$$
x_{1} \leq x_{2}, y_{2} \leq y_{1} \quad \Rightarrow \quad T\left(x_{1}, y_{1}\right) \leq T\left(x_{2}, y_{2}\right), T\left(y_{1}, x_{1}\right) \geq T\left(y_{2}, x_{2}\right),
$$

which means

$$
\left(x_{1}, y_{1}\right) \lesssim\left(x_{2}, y_{2}\right) \quad \Rightarrow \quad F\left(x_{1}, y_{1}\right) \lesssim F\left(x_{2}, y_{2}\right)
$$

i.e. $F$ is increasing.

Furthermore, by assumption, for any $x_{1}, x_{2}, y_{1}, y_{2} \in X, x_{1} \leq x_{2}, y_{2} \leq y_{1}$

$$
T\left(x_{2}, y_{2}\right)-T\left(x_{1}, y_{1}\right) \leq L\left(x_{2}-x_{1}\right)+S\left(y_{1}-y_{2}\right)=L\left(x_{2}-x_{1}\right)-S\left(y_{2}-y_{1}\right),
$$

then

$$
T\left(y_{1}, x_{1}\right)-T\left(y_{2}, x_{2}\right) \leq L\left(y_{1}-y_{2}\right)+S\left(x_{2}-x_{1}\right)
$$

or equivalently,

$$
T\left(y_{2}, x_{2}\right)-T\left(y_{1}, x_{1}\right) \geq-L\left(y_{1}-y_{2}\right)-S\left(x_{2}-x_{1}\right)=-S\left(x_{2}-x_{1}\right)+L\left(y_{2}-y_{1}\right),
$$

which implies

$$
F\left(x_{2}, y_{2}\right)-F\left(x_{1}, y_{1}\right) \lesssim \Phi\left[\left(x_{2}, y_{2}\right)-\left(x_{1}, y_{1}\right)\right], \quad \forall\left(x_{1}, y_{1}\right) \lesssim\left(x_{2}, y_{2}\right)
$$

where $\Phi: X \times X \rightarrow X \times X$ is defined as

$$
\Phi(x, y)=(L(x)-S(y),-S(x)+L(y)) .
$$

Note that

(1) $\Phi$ is a linear operator and $\Phi(P \times(-P)) \subset P \times(-P)$.

In fact, since $L, S$ is linear, $\Phi$ is a linear operator on $X \times X$.

If $(x, y) \in P \times(-P)$, i.e. $x \geq 0, y \leq 0$, due to the positivity of $L$, $S$, we have $L(x) \geq 0$,

$L(y) \leq 0, S(x) \geq 0, S(y) \leq 0$, then

$$
L(x)-S(y) \geq 0, \quad-S(x)+L(y) \leq 0
$$

i.e.

$$
\Phi(x, y) \in P \times(-P) .
$$

(2) $\Phi$ is bounded and its operator norm $\|\Phi\| \leq\|L\|+\|S\|<1$. 
In fact,

$$
\begin{aligned}
\|\Phi\| & =\sup _{(x, y) \in X \times X,\|(x, y)\|=1}\|\Phi(x, y)\| \\
& =\sup _{(x, y) \in X \times X,\|(x, y)\|=1} \max \{\|L(x)-S(y)\|,\|L(y)-S(x)\|\} \\
& \leq \sup _{(x, y) \in X \times X,\|(x, y)\|=1} \max \{\|L(x)\|+\|S(y)\|,\|L(y)\|+\|S(x)\|\} \\
& \leq \sup _{(x, y) \in X \times X, \max \{\|x\|,\|y\|\}=1} \max \{\|L\|\|x\|+\|S\|\|y\|,\|L\|\|y\|+\|S\|\|x\|\} \\
& \leq\|L\|+\|S\| .
\end{aligned}
$$

Then, by [4] Theorem 3.2, $F$ has a unique fixed point $(\bar{x}, \bar{y}) \in X \times X$. Moreover, for every $(x, y) \in X \times X$,

$$
\lim _{n \rightarrow \infty} F^{n}(x, y)=(\bar{x}, \bar{y})
$$

Hence, $T$ has a unique coupled fixed point $(\bar{x}, \bar{y})$ in $X \times X$. Moreover, for every $(x, y) \in$ $X \times X$,

$$
\lim _{n \rightarrow \infty} T^{n}(x, y)=\bar{x}, \quad \lim _{n \rightarrow \infty} T^{n}(y, x)=\bar{y} .
$$

This ends the proof.

Remark 4.3 If, in addition, the positive linear operators $L, S$ are commutative, i.e. $L S=S L$, then the requirement $\|L\|+\|S\|<1$ can be relaxed to $r(L)+r(S)<1$.

In this case, by mathematical induction, we have

$$
\Phi^{2 n}(x, y)=\left(\left(L^{2}+S^{2}\right)^{n}(x),\left(L^{2}+S^{2}\right)^{n}(y)\right), \quad \forall(x, y) \in X \times X,
$$

then

$$
\begin{aligned}
\left\|\Phi^{2 n}\right\| & =\sup _{(x, y) \in X \times X,\|(x, y)\|=1}\|\Phi(x, y)\| \\
& =\sup _{(x, y) \in X \times X,\|(x, y)\|=1} \max \left\{\left\|\left(L^{2}+S^{2}\right)^{n}(x)\right\|,\left\|\left(L^{2}+S^{2}\right)^{n}(y)\right\|\right\} \\
& \leq\left\|\left(L^{2}+S^{2}\right)^{n}\right\| .
\end{aligned}
$$

Hence, by Gelfand's formula

$$
\begin{aligned}
r(\Phi) & =\lim _{n \rightarrow \infty}\left\|(\Phi)^{n}\right\|^{\frac{1}{n}} \\
& =\lim _{n \rightarrow \infty}\left\|(\Phi)^{2 n}\right\|^{\frac{1}{2 n}} \\
& \leq \lim _{n \rightarrow \infty}\left\|\left(L^{2}+S^{2}\right)^{n}\right\|^{\frac{1}{2 n}} \\
& =\sqrt{r\left(L^{2}+S^{2}\right)}
\end{aligned}
$$




$$
\begin{aligned}
& \leq \sqrt{r(L)^{2}+r(S)^{2}} \\
& \leq r(L)+r(S) \\
& <1 .
\end{aligned}
$$

Then, by [4] Theorem 3.2, $F$ has a unique fixed point $(\bar{x}, \bar{y}) \in X \times X$.

Now, we extend the study to the multi-valued case.

In the same manner as the proof of Theorem 3.1 and Theorem 4.2, we can verify the following coupled fixed point theorem for multi-valued mixed monotone operators.

Theorem 4.4 Suppose $T: X \times X \rightarrow 2^{X} \backslash\{\phi\}$ is a multi-valued mixed monotone operator. Assume the following conditions are satisfied:

(1) For any $(x, y) \in X \times X, T(x, y)$ is a nonempty and closed subset of $X$.

(2) There exist two linear operators $L, S: X \rightarrow X,\|L\|+\|S\|<1, L(P) \subset P, S(P) \subset P$ such that, for any $x_{1}, x_{2}, y_{1}, y_{2} \in X, x_{1} \leq x_{2}, y_{2} \leq y_{1}$ imply:

(i) for any $u \in T\left(x_{1}, y_{1}\right)$, there exists $v \in T\left(x_{2}, y_{2}\right)$

$$
0 \leq v-u \leq L\left(x_{2}-x_{1}\right)+S\left(y_{1}-y_{2}\right)
$$

(ii) for any $v \in T\left(x_{2}, y_{2}\right)$, there exists $u \in T\left(x_{1}, y_{1}\right)$

$$
0 \leq v-u \leq L\left(x_{2}-x_{1}\right)+S\left(y_{1}-y_{2}\right)
$$

Then $T$ has a coupled fixed point in $X \times X$.

\section{Application to a fractional integral inclusion}

Let $(\Omega, \Sigma)$ be a measurable space and $X$ be a Banach space. We use the notations

$$
\begin{aligned}
& P_{f}(X)=\{A \subset X \mid A \text { is nonempty and closed }\}, \\
& P_{\text {kc }}(X)=\{A \subset X \mid A \text { is nonempty, compact and convex }\} .
\end{aligned}
$$

A multi-valued mapping $F: \Omega \rightarrow P_{f}(X)$ is said to be measurable if, for every $x \in X$,

$$
\omega \rightarrow d(x, F(\omega))=\inf _{z \in F(\omega)}\|x-z\|
$$

is measurable.

Let $X=C[0,1]$. For $x \in X$, let $\|x\|=\max _{t \in[0,1]}|x(t)|, P=\{x \in X \mid x(t) \geq 0, t \in[0,1]\}$, then $(X,\|\cdot\|)$ is a Banach space and $P$ is a normal and reproducing cone in $X$.

Let $0<\alpha<1$ be a constant. For $x \in X$, the Riemann-Liouville fractional integral operator $I^{\alpha}$ is defined as follows [27]:

$$
I^{\alpha} x(t)=\frac{1}{\Gamma(\alpha)} \int_{0}^{t}(t-s)^{\alpha-1} x(s) d s
$$


Let $f:[0,1] \times R \rightarrow 2^{R} \backslash\{\phi\}$ be a multi-valued function. For each $u \in X$, define the set of selections of $f$ by

$$
S_{f, u}=\left\{v \in I^{\alpha}: v(s) \in f(s, u(s)) \text { a.e. } s \in[0,1]\right\}
$$

where $I^{\alpha}$ stands for the collection of all Riemann-Liouville fractional integral functions, and further assume that $S_{f, u}$ is nonempty for each $u \in x$.

Now, let us consider the following fractional integral inclusion:

$$
x(t) \in \int_{0}^{t}(t-s)^{\alpha-1} f(s, x(s)) d s
$$

where

$$
\int_{0}^{t}(t-s)^{\alpha-1} f(s, x(s)) d s=\left\{\int_{0}^{t}(t-s)^{\alpha-1} u(s) d s: u(s) \in S_{f, x}\right\} .
$$

Theorem 5.1 Assume the following conditions hold:

(1) $f:[0,1] \times R \rightarrow 2^{R} \backslash\{\phi\}$ satisfies the following hypotheses:

(i) $f(t, x)$ in $P_{\mathrm{kc}}(R)$ for $(t, x) \in[0,1] \times R$;

(ii) for each $u \in X, t \rightarrow f(t, u(t))$ is measurable;

(iii) for each $t \in[0,1]$ and $u \in C[0,1], \sup _{x \in f(\cdot, u(\cdot))}\|x\| \in L^{1}$.

(2) There exists a constant $M>0$ such that for any $x, y \in X, x \leq y$ implies:

(i) for any $u \in \int_{0}^{t}(t-s)^{\alpha-1} f(s, x(s)) d s \cap C[0,1]$, there exists $v \in \int_{0}^{t}(t-s)^{\alpha-1} f(s, y(s)) d s \cap C[0,1]$ satisfying

$$
0 \leq v(t)-u(t) \leq \int_{0}^{t}(t-s)^{\alpha-1} M(y(s)-x(s)) d s,
$$

(ii) for any $v \in \int_{0}^{t}(t-s)^{\alpha-1} f(s, y(s)) d s \cap C[0,1]$, there exists $u \in \int_{0}^{t}(t-s)^{\alpha-1} f(s, x(s)) d s \cap C[0,1]$ satisfying

$$
0 \leq v(t)-u(t) \leq \int_{0}^{t}(t-s)^{\alpha-1} M(y(s)-x(s)) d s .
$$

Then the fractional integral inclusion has a solution in $C[0,1]$.

To prove the solvability of fractional integral inclusion, we need the following lemma.

Lemma 5.2 $I^{\alpha}$ is a linear, positive operator from $X$ to $X$. Moreover, the spectral radius $r\left(I^{\alpha}\right)=0$.

Proof The proof is given in two steps.

Step 1. $I^{\alpha}$ maps $X$ into $X$.

In fact, for arbitrary $u \in X$, assume $t, t+h \in[0,1]$. 
When $h>0$

$$
\begin{aligned}
\left|I^{\alpha} u(t+h)-I^{\alpha} u(t)\right|= & \mid \frac{1}{\Gamma(\alpha)} \int_{0}^{t+h}(t+h-\tau)^{\alpha-1} u(\tau) d \tau \\
& -\frac{1}{\Gamma(\alpha)} \int_{0}^{t}(t-\tau)^{\alpha-1} u(\tau) d \tau \mid \\
\leq & \frac{\|u\|}{\Gamma(\alpha)}\left|\int_{0}^{t}\left[(t+h-\tau)^{\alpha-1}-(t-\tau)^{\alpha-1}\right] d \tau\right| \\
& +\frac{\|u\|}{\Gamma(\alpha)}\left|\int_{0}^{t+h}(t+h-\tau)^{\alpha-1} d \tau\right| \\
= & \frac{\|u\|}{\Gamma(\alpha)}\left(\left|K_{1}\right|+\frac{h^{\alpha}}{\alpha}\right) .
\end{aligned}
$$

For $K_{1}$, we have the following estimation:

$$
\left|K_{1}\right|=\left|\int_{0}^{t}\left[(t+h-\tau)^{\alpha-1}-(t-\tau)^{\alpha-1}\right] d \tau\right|=h^{\alpha} \int_{0}^{\frac{t}{h}}\left[s^{\alpha-1}-(s+1)^{\alpha-1}\right] d s .
$$

(1) If $0 \leq t \leq h$,

$$
\left|K_{1}\right|<h^{\alpha} \int_{0}^{1}\left[s^{\alpha-1}-(s+1)^{\alpha-1}\right] d s<h^{\alpha} \frac{h^{\alpha}}{\alpha} .
$$

(2) If $t>h$, then

$$
\begin{aligned}
\left|K_{1}\right| & =\left|\frac{1}{\Gamma(\alpha)} \int_{0}^{t+h}(t+h-\tau)^{\alpha-1} u(\tau) d \tau-\frac{1}{\Gamma(\alpha)} \int_{0}^{t}(t-\tau)^{\alpha-1} u(\tau) d \tau\right| \\
& =h^{\alpha} \int_{0}^{1}\left[s^{\alpha-1}-(s+1)^{\alpha-1}\right] d s+h^{\alpha} \int_{1}^{\frac{t}{h}}\left[s^{\alpha-1}-(s+1)^{\alpha-1}\right] d s \\
& <\frac{h^{\alpha}}{\alpha}+(1-\alpha) h^{\alpha} \int_{1}^{\frac{t}{h}} s^{\alpha-2} d s \\
& <\frac{h^{\alpha}}{\alpha}+(1-\alpha) h^{\alpha} \int_{1}^{\infty} s^{\alpha-2} d s \\
& =\left(1+\frac{1}{\alpha}\right) h^{\alpha} .
\end{aligned}
$$

By (1), (2), we have

$$
\lim _{h \rightarrow 0}\left|I^{\alpha} u(t+h)-I^{\alpha} u(t)\right|=0 .
$$

In the same manner, we can prove $\lim _{h \rightarrow 0}\left|I^{\alpha} u(t+h)-I^{\alpha} u(t)\right|=0$ for $h<0$.

Hence, $I^{\alpha} u \in X$. The linearity and positivity of $I^{\alpha}$ are obvious.

Step 2. $r\left(I^{\alpha}\right)=0$.

By the semi-group property of the Riemann-Liouville fractional integral operator, we have

$$
\left(I^{\alpha}\right)^{n} u(t)=I^{n \alpha} u(t)=\frac{1}{\Gamma(n \alpha)} \int_{0}^{t}(t-\tau)^{n \alpha-1} u(\tau) d \tau
$$


hence

$$
\begin{aligned}
\left\|\left(I^{\alpha}\right)^{n}\right\| & =\sup _{\|u\|=1} \max _{0 \leq t \leq 1}\left|\frac{1}{\Gamma(n \alpha)} \int_{0}^{t}(t-\tau)^{n \alpha-1} u(\tau) d \tau\right| \\
& \leq \max _{0 \leq t \leq 1}\left|\frac{1}{\Gamma(n \alpha)} \int_{0}^{t}(t-\tau)^{n \alpha-1} d \tau\right| \\
& =\max _{0 \leq t \leq 1} \frac{1}{\Gamma(n \alpha)} \frac{1}{n \alpha} t^{n \alpha} \\
& \leq \frac{1}{\Gamma(n \alpha+1)}
\end{aligned}
$$

Due to Stiring's formula,

$$
\lim _{n \rightarrow \infty} \sqrt[n]{\Gamma(n \alpha+1)}=\lim _{n \rightarrow \infty} \sqrt[n]{\left(\frac{n \alpha}{e}\right)^{n \alpha} \sqrt{2 n \pi \alpha}(1+o(1))}=\lim _{n \rightarrow \infty}\left(\frac{n \alpha}{e}\right)^{\alpha}=\infty .
$$

By Gelfand's formula,

$$
r\left(I^{\alpha}\right)=\lim _{n \rightarrow \infty}\left\|\left(I^{\alpha}\right)^{n}\right\|^{\frac{1}{n}} \leq \lim _{n \rightarrow \infty}\left|\frac{1}{\sqrt[n]{\Gamma(n \alpha+1)}}\right|=0 .
$$

Then $r\left(I^{\alpha}\right)=0$.

This ends the proof.

Proof of Theorem 5.1 Define a multi-valued mapping $T$ from $X$ to $2^{X} \backslash\{\phi\}$ as follows:

$$
T(x)(t)=\int_{0}^{t}(t-s)^{\alpha-1} f(s, x(s)) d s, \quad x \in X .
$$

From assumption (1) we know that $T$ has nonempty values. Because of the Rädstrom embedding theorem [28], it is easy to show

$$
T(x)(t) \in P_{\mathrm{kc}}(R), \quad t \in[0,1] .
$$

Then an application of Arzela and Ascoli's theorem shows that $T$ has values in $P_{\mathrm{kc}}(C[0,1])$. Assumption (2) means there exists a linear operator $L: X \rightarrow X$ such that, for any $x, y \in X$, $x \leq y$ implies:

(i) for any $u \in T(x)$, there exists $v \in T(y)$ satisfying

$$
0 \leq v-u \leq L(y-x)
$$

(ii) for any $v \in T(y)$, there exists $u \in T(x)$ satisfying

$$
0 \leq v-u \leq L(y-x)
$$

where $L(x)(t)=M \int_{0}^{t}(t-s)^{\alpha-1} x(s) d s$. By Lemma 5.2, $r(L)=0$ and $L(P) \subset P$. Due to Theorem 3.1, $T$ has a fixed point in $x$, i.e., the fractional differential inclusion has a solution.

This ends the proof. 


\section{Competing interests}

The authors declare that they have no competing interests.

\section{Authors' contributions}

All authors contributed equally to the writing of this paper. All authors read and approved the final manuscript.

\section{Acknowledgements}

We are grateful to the anonymous referees for their helpful suggestions. This research is supported in part by the Doctoral Fund of Education Ministry of China (20134219120003) and the Key Program of Natural Science Foundation of China (71231007).

Received: 5 February 2016 Accepted: 18 May 2016 Published online: 27 May 2016

\section{References}

1. Amann, H: Fixed point equations and nonlinear eigenvalue problems in ordered Banach spaces. SIAM Rev. 18 , 620-709 (1976)

2. Heikkilä, S, Lakskmikantham, V: Monotone Iterative Techniques for Discontinuous Nonlinear Differential Equations. Dekker, New York (1994)

3. Guo, D: Partial Order Methods in Nonlinear Analysis. Shandong Science and Technology Press, Jinan (2000)

4. Feng, Y, Wang, H: Characterizations of reproducing cone and uniqueness of fixed point. Nonlinear Anal. 74, 5759-5765 (2011)

5. Guo, D, Lakskmikantham, V: Coupled fixed points of nonlinear operators with applications. Nonlinear Anal. 11, 623-632 (1987)

6. Bhaskar, TG, Lakskmikantham, V: Fixed point theory in partially ordered metric spaces and applications. Nonlinear Anal. 65, 1379-1393 (2006)

7. Guo, D: Existence and uniqueness of positive fixed point for mixed monotone operators with applications. Appl. Anal. 46, 91-100 (1992)

8. Zhang, Z: New fixed point theorems of mixed monotone operators and applications. J. Math. Anal. Appl. 204 307-319 (1996)

9. Zhang, S, Ma, Y: Coupled fixed points for mixed monotone condensing operators and an existence theorem of the solution for a class of functional equations arising in dynamic programming. J. Math. Anal. Appl. 160, 468-479 (1991)

10. Sun, Y: A fixed point theorem for mixed monotone operator with applications. J. Math. Anal. Appl. 156, 240-252 (1991)

11. Lian, $X, \mathrm{Li}$, Y: Fixed point theorems for a class of mixed monotone operators with applications. Nonlinear Anal. 67 , 2752-2762 (2007)

12. $\mathrm{Wu}, \mathrm{Y}, \mathrm{Liang}, \mathrm{Z}$ : Existence and uniqueness of fixed points for mixed monotone operators with applications. Nonlinear Anal. 65, 1913-1924 (2006)

13. Kukushikin, NS: A fixed point theorem for decreasing mappings. Econ. Lett. 46, 23-26 (1994)

14. Harjani, J, Lopez, B, Sadarangani, K: Fixed point theorems for mixed monotone operators and applications to integral equations. Nonlinear Anal. 74, 1749-1760 (2011)

15. Bu, C, Feng, Y, Li, H: Existence and uniqueness of fixed point for mixed monotone ternary operators. Fixed Point Theory Appl. 2014, 223 (2014)

16. Nishniannidz, ZG: Fixed points of monotonic multiple-valued operators. Bull. Georgian Acad. Sci. 114, $489-491$ (1984)

17. Nguyen, BH, Nguyen, HK: Fixed point for multivalued increasing operators. J. Math. Anal. Appl. 250, 368-371 (2000)

18. Nguyen, $\mathrm{BH}$ : Fixed point of increasing multivalued operators and an application to the discontinuous elliptic equations. Nonlinear Anal. 51,673-678 (2002)

19. Huang, N, Fang, Y: Fixed points for multi-valued mixed increasing operators in ordered Banach spaces with applications to integral inclusions. Z. Anal. Anwend. 22, 399-410 (2003)

20. Feng, Y, Liu, S: Fixed point theorems for multi-valued increasing operators in partial ordered spaces. Soochow J. Math 30, 461-469 (2004)

21. Hong, S: Fixed points for mixed monotone multivalued operators in Banach space with applications. J. Math. Anal. Appl. 337, 333-342 (2008)

22. Hong, S: Fixed points of multivalued operators in ordered metric spaces with applications. Nonlinear Anal. 72 3929-3942 (2010)

23. Hong, S, Qiu, Z: Fixed points of discontinuous multivalued operators in ordered spaces with applications. Fixed Point Theory Appl. 2010, Article ID 745769 (2010). doi:10.1155/2010/745769

24. Feng, $Y$, Tong, P: Existence and nonexistence of positive periodic solutions to a second order differential inclusion. Topol. Methods Nonlinear Anal. 42, 449-459 (2013)

25. Feng, Y, Mao, W, Qu, G: Fixed point theorems of multi-valued decreasing operators on cones. J. Nonlinear Sci. Appl. 8, 334-339 (2015)

26. Krasnoses'kii, MA, Zabreiko, PP: Geometrical Methods of Nonlinear Analysis. Springer, Berlin (1984)

27. Diethelm, K: The Analysis of Fractional Differentiations: An Application-Oriented Exposition Using Differential Operators of Caputo Type. Springer, Berlin (2010)

28. Klein, E, Thompson, A: Theory of Correspondences. Wiley, New York (1984) 